\title{
Clinical Profile, Haematological Changes and Outcomes of Dengue Patients in Dengue Outbreak-2019 in Jashore, Bangladesh- An Observational Study
}

\author{
Mohammad Ali ${ }^{1}$, Goutam Kumar Acherjya², AKM Mynul Islam³ ${ }^{3}$ ABM Saiful Alam4, SM Shafiqur Rahman ${ }^{5}$, \\ Ranajit Sen Chowdhury ${ }^{6}$, Md Shamsuzzaman7, Rajashish Chakrabortty ${ }^{8}$, Gobinda Chandra Roy ${ }^{9}$
}

\begin{abstract}
:
Background: Bangladesh is an endemic zone for re-emerging dengue viral infection since 2000. For the last few years, dengue viral outbreak occurs in the rainy season mostly in Dhaka city and seldom sporadic case finds beyond the Dhaka city of Bangladesh. But this outbreak expands also outside the Dhaka city of Bangladesh in 2019. Therefore, with scarcity of data regarding this field in the peripheral part of Bangladesh, we have designed this study among dengue infected patients in Jashore, Bangladesh.

Objectives: This observational study aims to assess the clinical presentations, laboratory findings especially hematological changes and outcomes of patients with dengue viral infection at Jashore district in dengue outbreak-2019 in Bangladesh.

Methodology: This was an observational study, carried out from $25^{\text {th }}$ April 2019 to $5^{\text {th }}$ September 2019 during dengue outbreak season in Jashore, Bangladesh. Patients were selected from outpatient department and indoor of a local private hospital in Jashore, Bangladesh who had given informed written consent to participate in our study.

Result: Total eligible 77 patients were included in this study. Out of which 70 patients (90.9\%) were dengue NSI antigen Positive on Immunochromatography (ICT) and the rest were anti-dengue IgM antibody positive on ICT method. Around 74 (96.l\%) patients had fever as the most common feature in dengue infection. Eighty-seven percentages (87\%) of patients had history of headache. Other striking complaints were body ache (66.2\%), back pain (5I.9\%), nausea (55.8\%), vomiting (3I.2\%), anorexia (27.3\%), arthralgia (29.9\%) and retro-orbital pain (26.0\%). Sore throat being a common feature in flu-like illness also found in $18.2 \%$ cases. Mean total count of whole blood count (WBC) falls on day $5\left(5.76 \times 10^{9} / \mathrm{L}\right)$ and then gradually increased. Mean platelet count of study patient was normal throughout the course of the disease process. But, in some cases minimum platelets was found $21 \times 10^{9} / \mathrm{L}$ at the time of presentation and on day 5 and day 6 which was dropped down to $17 \times 10^{\%} / \mathrm{L}$. Mean Haematocrit (\%) was more or less in a steady state throughout the acute phage of the disease. But, in some cases throughout the hospital stay minimum haematocrit was less than $30 \%$ and initially in some cases maximum haematocrit was more than $50 \%$ among some dengue infected patients. Total 67 (87\%) subjects with dengue viral infection required hospital admission and mean hospital stay was 4 days with a standard deviation (SD) was \pm 2 days. Blood transfusion was required in only one patient. Around $95 \%$ patient was recovered and only 4 cases were referred to the higher centre further better management. Regarding complications, we had found diarrhoea in $9(11.7 \%)$ and bleeding disorder in $14.3 \%$ patients.
\end{abstract}

1. Assistant Professor (Haematology), National Institute of Cancer Research and Hospital, Mohakhali, Dhaka, Bangladesh.

2. Assistant Professor (Medicine), Jashore Medical College, Jashore, Bangladesh.

3. Assistant Professor (Haematology), National Institute of Cancer Research and Hospital, Mohakhali, Dhaka, Bangladesh.

4. Associate Professor (Medicine), Jashore Medical College, Jashore, Bangladesh.

5. Assistant Professor (Neurology), Jashore Medical College, Jashore, Bangladesh.

6. Associate professor (Medicine), Sir Salimullah Medical College Mitford Hospital, Dhaka, Bangladesh.

7. Specialty Doctor in Acute Medicine, Leighton Hospital, Mid Cheshire NHS Foundation Trust, Crewe, United Kingdom.

8. Associate Professor (Respiratory Medicine), Bangabandhu Sheikh Mujib Medical University, Dhaka, Bangladesh.

9. Associate professor (Medicine), Shaheed Suhrawardy Medical College, Dhaka, Bangladesh.

Correspondence: Goutam Kumar Acherjya, Assistant Professor, (Medicine), Jashore Medical College, Jashore, Bangladesh, email: gacherjya@hotmail.com 
Conclusion: In our study, the most common clinical presentations were fever, headache, body-ache, back pain, and gastrointestinal upset. The mean hematological started dropping down from $4^{\text {th }}$ day and gradually improved after $7^{\text {th }}$ day. Some patients developed diarrhea, bleeding disorders and hepatitis as complications during their disease process. We observed a good clinical outcome evident by without having severity and death toll among our study participants. There was very seldom requirement of blood and blood product transfusion in our study.

Keywords: Dengue Viral Infections, Case detection, Common manifestations, Haematological changes, Complications, Treatment outcome.

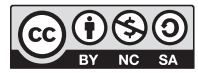

DOI: https://doi.org//0.3329/jom.v22il.5/389

Copyright: (C) 202I Ali M. This is an open access article published under the Creative Commons AttributionNonCommercial-NoDerivatives 4.0 International License, which permits use, distribution and reproduction in any medium, provided the original work is properly cited, is not changed in any way and it is not used for commercial purposes.

Received: 07 November, 2020;

Accepted: 28 November, 2020

\section{Introduction}

Dengue is a mosquito-borne viral disease caused by dengue virus belonging to Flaviviridae especially endemic in the tropical and sub-tropical regions of the world. ${ }^{1}$ This tropical disease is transmitted by Aedes aegypti mosquito with an incubation period of 4-7 days ranging from 3 to 14 days. $^{2}$ This acute self-limiting systemic viral infection can be caused by any of the four serotypes including DENV-1, DENV-2, DENV-3 and DENV-4. So, a person has a chance to be infected four times in his life as infection with one serotype of DENV providing lifelong immunity to the specific serotype. Genetically dengue serotypes are closely related to each other but they are antigenically distinct from each other. ${ }^{1}$ The dengue viral infection has varieties of clinical presentations according to the severity such as asymptomatic disease, classical dengue fever (DF), dengue hemorrhagic fever (DHF), dengue shock syndrome (DSS) and expended dengue syndrome (EDS). Additionally, potential risk of development of dengue severity with subsequent different dengue serotype infection. ${ }^{1,3}$ According to the World Health Organization (WHO) dengue is now transmitted every parts of the WHO region especially tropical and subtropical countries; and it is accounted as an endemic in 125 countries including Bangladesh. The global incidence of dengue infection per year is estimated approximately 400 million people. Factors such as modern dynamics of climate change, globalization, socioeconomics settlement, and viral evolution are thought to be the responsible for expansion of dengue viral infection. ${ }^{4}$ Though severe form of dengue may lead to serious illness and even death in a significant number of the patients; and there is no specific treatment for dengue, so therefore, early detection and prompt medical care can lower the case fatality and severity of dengue infection. Moreover, dengue outbreak has been reported in many Latin American and Asian countries including Bangladesh over the last couple of decades. We had noticed the first dengue outbreak in 2000 in Bangladesh, since then the case number per year was minimal, but it exceeded all the previous record in the year of 2019. More than hundred thousand cases were documented according to the government record in 2019 dengue outbreak but which might be higher than the actual record. ${ }^{5,6}$ During the last outbreak dengue cases were initially confined to the capital city of Bangladesh but soon it spread all over country which was an exceptional event this year. With usual clinical spectrum, a number of atypical presentation including increased vascular fragility and permeability, gastrointestinal symptoms, ascites, splenomegaly, pneumonia, pleural effusion, neurological manifestations (encephalitis, encephalopathy, and GuillainBarre syndrome), pericarditis and myocarditis also reported in 2019 dengue outbreak. $^{7}$

During this dengue outbreak, a number of physicians especially who were working beyond the capital city of Bangladesh experienced some new challenges in term of a wide spectrum atypical clinical presentations of dengue. In addition, dengue was previously confined to the capital city of Bangladesh; there was scarcity of dengue related data in the peripheral part of the country like Bangladesh. Hence, with this view we have designed this study to assess the clinical presentations, laboratory findings especially hematological changes and outcome of conservative management of patients with dengue viral infection in Jashore, Bangladesh. 


\section{Methods}

This observational study was carried out from $25^{\text {th }}$ April 2019 to $05^{\text {th }}$ September 2019 during dengue outbreak season in 2019 at Jashore, Bangladesh. Patient were selected form outpatient department and indoor of Modern Diagnostic Centre and Hospital, Jashore, Bangladesh. A preformed structured questionnaire was developed to collect data. All the Dengue cases 15 years or above who were confirmed by Immunochromatography test (ICT) for dengue $\mathrm{NS}_{1}$ or Anti dengue IgM antibody positivity enrolled in our study. For the haematological assessment, patients who attended 4 days after symptomatic manifestations were excluded from our study. Initially patients were enrolled in outpatient services after serological positivity and cases were admitted according to clinical severity. Complete blood count (CBC) was sent on the day of admission or consultation and at day 5 onwards. Three cc of blood sample was drawn from each participant after enrolment which was mixed with EDTA for Complete Blood count $(\mathrm{CBC})$ and carried out in auto cell analysers, i.e., Sysmex XN550. A blood smear was stained with Giemsa stain, and a differential count was performed to determine the percentages of normal Neutrophils, Lymphocytes, and Monocytes. Platelet counts were recorded in each individual and the results were verified on slide. Other clinical investigations were done according to the patients' clinical condition. Clinical presentations, complications and requirement of blood and platelet transfusion were documented up-to recovery or discharge of the patients from hospital. Patients were discharged once being clinically asymptomatic, haematologically and biochemically stable. Total duration of hospital stay and outcome of treatment were also documented in our study.

\section{Ethical Approval}

Institutional approval was taken from Jashore Medical College and Hospital; and informed written consent was taken from the each participant prior to collect the data. The data would be used only for research purpose and it was also assured that any of the patients' data won't be handed over to any of the third party at any quest.

\section{Statistical analysis}

Continuous data was grouped as mean and standard deviation (SD) and categorical data were presented as number and percentages (\%). SPSS version 23 was employed for statistical analysis.

\section{Result}

Total 77 cases of dengue confirmed patients by Immunochromatographic test of dengue NS1 and anti dengue IgM antibody were enrolled in this study. Among them 33 were male and rest 44 cases were female with a male-female ratio was 1:0.75. Mean age of study subjects were 37 years with a standard deviation of 14 years. (Table I).

In this study group of patients, there was no known history of previous dengue infection but anti dengue $\mathrm{IgG}$ was found positive in $27(35.1 \%)$ patients. Co-existing conditions like hypertension, ischemic heart disease, diabetes mellitus, bronchial asthma or chronic obstructive pulmonary disease (COPD) were found in $35.1 \%$ of dengue patients in our study. Around $90 \%$ cases were confirmed by immunochromatography test (ICT) dengue NS1 and rest 7 was confirmed by ICT anti dengue IgM antibody positivity. (Table II).

Around 74 (96.1\%) patients were presented with fever and next to it, eighty-seven percentages of patients noticed headache. Other prominent complaints were body ache (66.2\%), back-pain $(51.9 \%)$, nausea $(55.8 \%)$, vomiting $(31.2 \%)$, anorexia $(27.3 \%)$, arthralgia $(29.9 \%)$ and retroorbital pain (26.0\%). Only 4 patients $(5.2 \%)$ had rash and 9 $(11.7 \%)$ patient had hepatomegaly on abdominal palpation. (Table III).

Table 1

Age and sex distribution of study patients- (n: 77)

\begin{tabular}{llccc}
\hline Characteristics & Mean \pm SD & Min-Max & Median & $\mathrm{n}(\%)$ \\
\hline Age of patients in years- & $37 \pm 14$ & $15-73$ & 36 & $77(100)$ \\
Sex- & \multicolumn{4}{c}{ Male: Female $=0.75$} \\
$\quad$ Male & & $33(42.9)$ \\
$\quad$ Female & & $44(57.1)$ \\
\hline
\end{tabular}

Min: minimum, Max: maximum, n: number, \%: percentage, SD: Standard deviation. 
Table-II

Patient characteristics and diagnosis - (n: 77)

\begin{tabular}{lc}
\hline Characteristics & $\mathrm{n}(\%)$ \\
\hline History of travel to Dhaka during & $6(7.8)$ \\
dengue outbreak- & $0(0.0)$ \\
$\begin{array}{l}\text { Previous history of confirmed dengue fever- } \\
\text { co-existing condition- }\end{array}$ & $0(0.0)$ \\
Pregnancy & $3(3.9)$ \\
Diabetes mellites & $12(15.6)$ \\
Hypertension & $3(3.9)$ \\
Ischemic heart disease & $9(11.7)$ \\
Bronchial asthma or COPD & \\
Diagnosis confirmed by- & $70(90.9)$ \\
ICT-positive dengue NS & \\
ICT-positive anti-dengue IgM & $7(9.1)$ \\
ICT-positive anti-dengue IgG & 35.1 \\
\hline
\end{tabular}

n: number, \%: percentage.

\section{Table-III}

Presentations of Dengue in the study groups. (n:77)

\begin{tabular}{lc}
\hline Clinical Presentations & $\mathrm{n}(\%)$ \\
\hline Fever & $74(96.1)$ \\
Headache & $67(87.0)$ \\
Body ache & $51(66.2)$ \\
Nausea & $43(55.8)$ \\
Backpain & $40(51.9)$ \\
Vomiting & $24(31.2)$ \\
Anorexia & $21(27.3)$ \\
Retro-orbital pain & $20(26.0)$ \\
Sore Throat & $14(18.2)$ \\
Photophobia & $7(9.1)$ \\
Rash & $4(5.2)$ \\
Arthralgia & $23(29.9)$ \\
Myalgia & $3(3.9)$ \\
Abdominal Pain & $6(7.8)$ \\
Hepatomegaly & $9(11.7)$ \\
\hline
\end{tabular}

n: number, \%: percentage

We had documented total WBC count, total platelets count, haematocrit, absolute neutrophil count, absolute lymphocyte count, and absolute monocyte count at the time of diagnosis or from day of consultation or admission or $4^{\text {th }}$ day of symptom presentation which one appeared first and ended up to the day 8. (Figure 1)

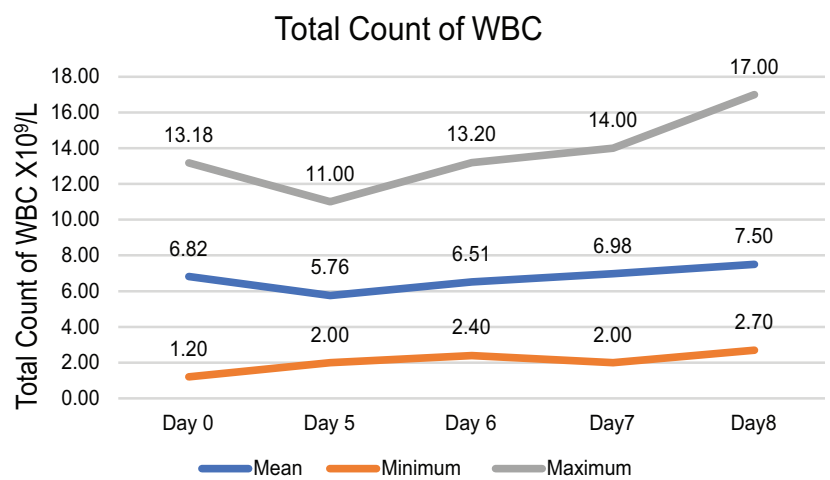

Fig.1: Mean, Minimum and Maximum total count of WBC at diagnosis and day 5 to day8 in dengue patients.

Mean total count of WBC was fallen after $4^{\text {th }}$ day $\left(5.76\right.$ X $10^{9} /$ $\mathrm{L}$ ) and then it gradually increased after $7^{\text {th }}$ day onwards. In some cases, total count of WBC was as low as $1.2 \mathrm{X} 10^{9} / \mathrm{L}$ at the time of presentation and then gradually increased. (Figure1)

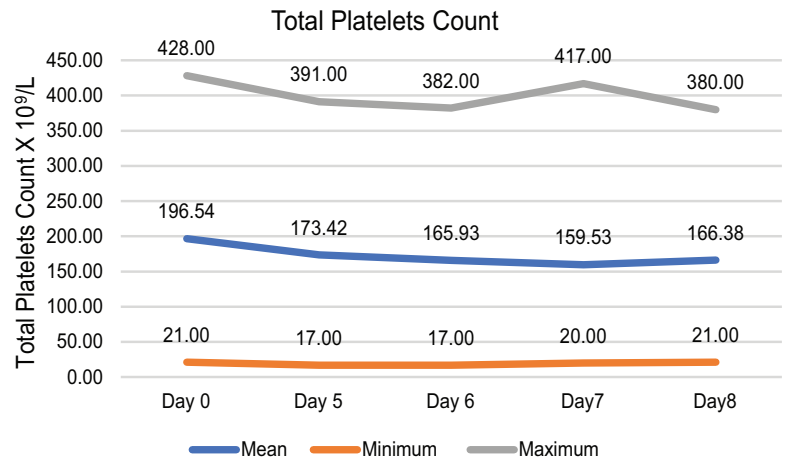

Figure 2: Mean, Minimum and Maximum total platelet count at diagnosis and day 5 to day 8 in dengue patients.

In our observation, mean platelet count of study patient was normal. But in some cases minimum platelets was found 21 $\mathrm{X} 10^{9} / \mathrm{L}$ at the time of presentation and on Day 5 and day 6 it was dropped down as low as $17 \mathrm{X} 10^{9} / \mathrm{L}$. It gradually appeared to be within normal limit from day 7 to onwards. (Figure: 2)

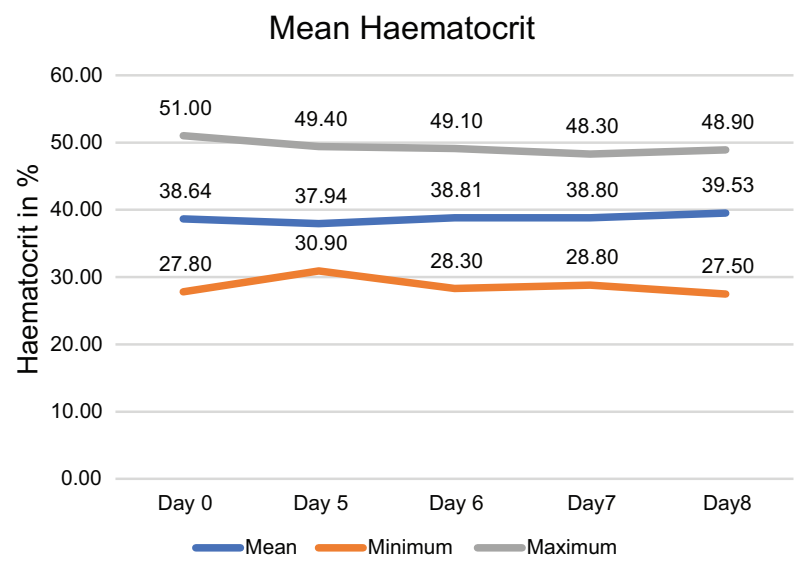

Figure 3: Mean, Minimum and Maximum Haematocrit in $\%$ at diagnosis and day 5 to day 8 in dengue patients. 
In our observation, mean Haematocrit level (in \%) was more or less in the steady state. But in some cases, the minimum haematocrit was less than $30 \%$ and initially in some cases maximum haematocrit was found more than $50 \%$ throughout the hospital stay. (Figure 3)

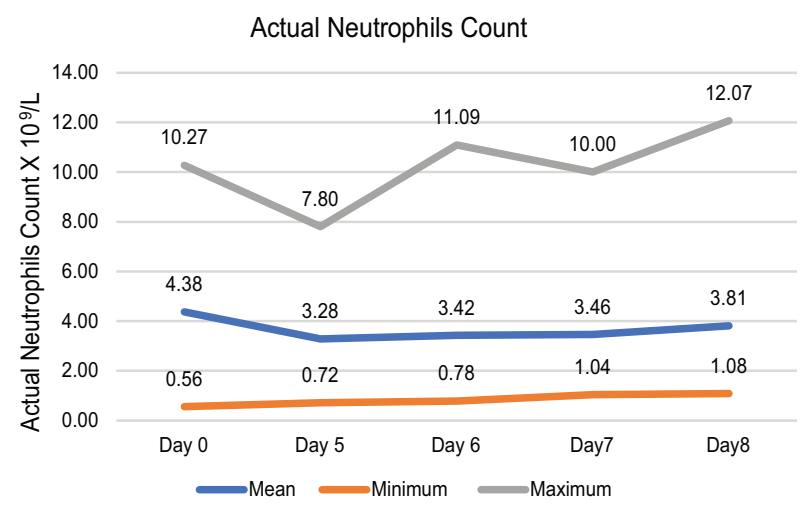

Figure 4: Mean, Minimum and Maximum total Neutrophil count at diagnosis and day 5 to day 8 in dengue patients.

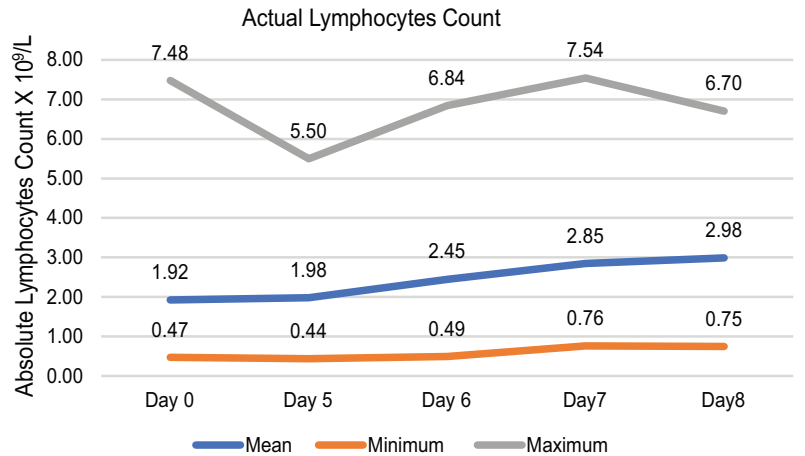

Figure 5: Mean, Minimum and Maximum total Lymphocytes count at diagnosis and day 5 to day 8 in dengue patients.
Absolute neutrophils count (ANC) had fallen during treatment then gradually it rose in our observation. But in some cases, ANC dropped down less than 01 X10 $10^{9}$ L initially but it was gradually raised. (Figure 4)

Mean absolute lymphocytes count and mean absolute monocytes counts were in the steady manner in our observation but in some cases minimum absolute lymphocytes count was less than $0.5 \times 10^{9} / \mathrm{L}$ at the time of presentation. (Figure 5, 6)

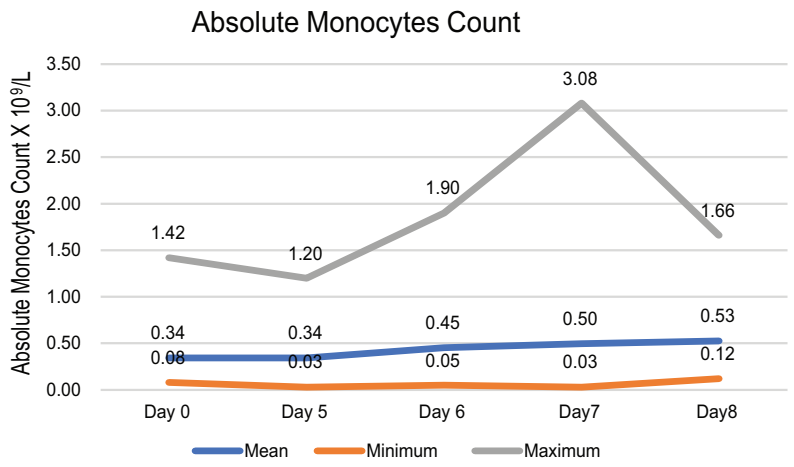

Figure 6: Mean, Minimum and Maximum total Monocytes count at diagnosis and day 5 to day 8 in dengue patients.

In our study, total 67 (87\%) subjects with dengue viral infection required hospital admission and mean hospital stay was 4 days with a standard deviation (SD) was \pm 2 days. Blood transfusion was required in only one patient who developed severe per vaginal bleeding despite of normal platelet count which was managed conventionally. Around $95 \%$ patients were recovered in this low resources health care centre, whereas, only 4 cases were referred to the higher centre for better management. We have found some complications like diarrhoea (11.7\%), gum bleeding (6.55\%), and per-vaginal bleeding (3.9\%) which were non-fatal in nature. (Table 4)

Table-IV

Hospital stay, outcome after treatment and complications during management. (n:77)

\begin{tabular}{|c|c|c|c|}
\hline Characteristics & Mean \pm SD & Min-Max & $\mathrm{n}(\%)$ \\
\hline Required admission & & & $67(87)$ \\
\hline Duration of hospital stay in days & $4 \pm 2$ & $1-8$ & \\
\hline Number of patients required blood transfusion & & & $1(1.3)$ \\
\hline Number patients required platelets concentrate transfusion & & & $0(0.0)$ \\
\hline \multicolumn{4}{|l|}{ Treatment outcome } \\
\hline - $\quad$ Recovery & & & $73(94.8)$ \\
\hline - $\quad$ Referred to higher centre & & & $4(5.2)$ \\
\hline - Death & & & $0(0.0)$ \\
\hline \multicolumn{4}{|l|}{ Complication encountered } \\
\hline - Diarrhoea & & & $9(11.7)$ \\
\hline - $\quad$ Gum bleeding & & & $5(6.5)$ \\
\hline - $\quad$ Per-vaginal bleeding & & & $3(3.9)$ \\
\hline - Malena & & & $2(2.6)$ \\
\hline - $\quad$ Epistaxis & & & $1(1.3)$ \\
\hline - Hepatitis & & & $1(1.3)$ \\
\hline
\end{tabular}

Min: minimum, Max: maximum, n: number, \%: percentage, SD: Standard deviation. 


\section{Discussion:}

Dengue shares a common clinical features like other viral infections, therefore, polymerase chain reaction (PCR) or non-structural (NS)1 antigen of dengue is required to diagnose within the early few days of dengue infection. But this can't differentiate the dengue disease severity either DF to DHF/DSS. However, the severity of dengue infection can be demonstrated by clinical, hematological and biochemical change of the infected patients. Moreover early detection of plasma leakage features within the dengue affected patients may help to reduce the progression of disease severity and potential risk of morbidity and mortality. ${ }^{8}$ The mean age of our study population was $37 \pm 14$ years with male and female ratio of 0.75 . There was no previous known or documented history of confirmed dengue fever previously, but only $7.8 \%$ of the study subjects had travel history to Dhaka one month prior to be infected during dengue outbreak, 2019. Underlying disease conditions were well considered during dengue case management as these conditions might interfere with fluid management and dictate the development of overwhelming further complications. Out of the total study subjects we had found the following preexisting diseases as hypertension $15.6 \%$, bronchial asthma or chronic obstructive pulmonary disease $11.7 \%$, diabetes mellitus $3.9 \%$ and coronary heart diseases $3.9 \%$. In addition, long term preexisting medical conditions might be a potential risk for development of severe dengue diseases, so earlier triage of these conditions might helpful for case management and reduce dengue morbidity and mortality. ${ }^{9,10}$ We had included only acute infected dengue patients evidenced by NS1 antigen (90.9\%) and ICT-positive anti-dengue IgM antibody $(9.1 \%)$.

Like other studies we had found that fever (96.1\%), headache $(87.0 \%)$, body-ache $(66.2 \%)$, back pain $(51.9 \%)$ and abdominal symptoms were most commonly reported symptoms among the dengue infected patients in our study. ${ }^{11-}$

${ }^{15}$ Sore throat was usually absent among the dengue patients in our country ${ }^{14,15}$ but we had noticed it in $18.2 \%$ of our study subjects. Sore throat is being common feature in flu like illness, but this uncommon symptom was also reported among the dengue patients in other studies like us. ${ }^{11,16}$ Hemorrhagic manifestations as unusual bleeding from various mucosal sites were common complications in dengue patients due to reduced platelet count and leakage from the blood vessels. The mechanism of thrombocytopenia in dengue patients are complex involving platelet activation, activation of pro-coagulant and anticoagulant arms of the coagulation system, complement, cytokines, and endothelial cells. ${ }^{17}$ Gum bleeding (6.5\%) was the most common form in our study whereas epistaxis had reported as commonest form in another study from Bangladesh. ${ }^{16}$ We had found other form bleeding manifestations as per-vaginal bleeding $(3.9 \%)$, melaena $(2.6 \%)$, and epistaxis $(1.3 \%)$ in our study. Hepatic involvement was commonly evident in dengue infection including hepatomegaly, raised serum bilirubin and hepatic enzymes. We had found hepatomegaly during clinical examination in $11.7 \%$ of study subjects, similarly with other study reported $14.8 \%$ of hepatomegaly. ${ }^{18}$ diarrhea was evident in $11.7 \%$ of our study subjects which is less frequent in another study from Bangladesh. ${ }^{14}$ In our study $87 \%$ of the patients required hospital admission due to different reasons including systemic upset, reduced platelet count, and familial influence. Mean duration of hospital stay of our study subjects was $4 \pm 2$ days; the duration of hospital stay was higher as $6 \pm 1$ day in one of the prospective follow up study of Sri Lanka. ${ }^{8}$

Sequential haematological changes of our study subjects recorded from the day of admission or $4^{\text {th }}$ day of dengue symptoms presentation which appeared first and onward up to day 8 . Total white blood count level was approximate $1.2 \times 10^{9} / \mathrm{L}$ at time of their presentation before day 5 , whereas mean total count was $5.76 \times 10^{9} / \mathrm{L}$ on day 5 of their presentation. The count gradually increased $6^{\text {th }}$ day onward of their presentation. A study from the Northern India reported the same finding like us. ${ }^{16}$

Thrombocytopenia is another common presentation that can be found at any stage of dengue infected patients. Moreover thrombocytopenia is thought to be responsible for one of the major causes of bleeding in dengue infection. ${ }^{19}$ Though the mean platelet count of the enrolled patients was more or less normal throughout the disease process, but some patients presented with low platelet count as low as $21 \mathrm{X} 10^{9} / \mathrm{L}$ at the time of presentation. We had noticed the lowest platelet count as $17 \times 10^{9} / \mathrm{L}$ on day 5 and 6 of their presentation in some patients. This was reported by the other study where the authors had found lowest plate count on day 4.5 to 6 among their cohorts. ${ }^{8}$ We had also noticed rising platelet count from the day 7 onward, this due to spontaneous decrease in platelet activation markers which was supported by other studies. ${ }^{8,19}$ Kinetic changes in absolute monocyte and lymphocyte count occurred along with thrombocytopenia which increased gradually on day 7 or onward in our study which is consistent with other study. ${ }^{21}$

One of our patients required blood transfusion due to profuse per vaginal bleeding despite of normal platelet count during her acute phage of the dengue infection. Whereas, we did not used pooled platelet transfusion in any of our subject. However, the recovery rate $(94.8 \%)$ with conventional treatment was also highly satisfactory in our setting. We had 
referred only 4 patients to higher centre for better management, three patients of whom developed, of note, altered conscious level during their disease course. One of them, who developed persistent thrombocytopenia even after extensive evaluation, was referred for further haematological evaluation.

\section{Conclusion:}

Dengue infection, as a multisystem disorder, atypical manifestations and life-threatening complications during disease process should be screened along with its usual presentations. Fever, headache, body ache and upper gastrointestinal symptoms are the most common clinical features in our study. The mean hematological deteriorated from $4^{\text {th }}$ day and gradually improved from $7^{\text {th }}$ day onwards. Blood and blood product have a very minimal role in our study with satisfactory outcomes evident by without any death toll. So early triage, extensive evaluation and supportive management can reduce the dengue morbidity and mortality.

\section{Limitation and Recommendations:}

This single center observational study has conducted on small sized sample. Even we could not extensively evaluate and investigate the patients due lack of funding. So, we recommend conducting a multi-center large scale study in Bangladesh.

Conflict of Interest: The authors have declared no conflict of interest.

Funding Sources: We had not obtained any fund to complete this manuscript.

\section{References:}

1. World Health Organization. WHO Report on Global Surveillance of Epidemic-prone Infectious Diseases. Geneva: World Health Organization, Department of Communicable Disease Surveillance; 2000 Dengue and Dengue Haemorrhagic Fever and Response; 2000 [cited 07 May 2020];[Chapter7].Availablefrom:https://www.who. int/csr/ resources/publications/surveillance WHO_CDS_CSR_ISR_2000_1/en/

2. World Health Organization. Fact sheet $N^{\circ} 117$. Dengue and Dengue haemorrhagic fever. Geneva: World Health Organization;2002 [cited 07 May 2020]. Available from: http:/www. who. int/mediacentre/factsheets/fs117/en/

3. World Health Organization. Comprehensive guideline for prevention and control of dengue and dengue haemorrhagic fever. India: World Health Organization, Regional Office for South-East Asia; 2011[cited 07 May 2020]. Available from: https://apps.who.int/iris/handle/10665/204894.
4. Murray NE, Quam MB, Wilder-Smith A. Epidemiology of dengue: past, present and future prospects. Clinical epidemiology. 2013;5:299. doi: 10.2147/CLEP.S34440

5. Mamun MA, Misti JM, Griffiths MD, Gozal D. The dengue epidemic in Bangladesh: risk factors and actionable items. The Lancet. 2019 Dec 14;394(10215):2149-50. DOI: https:/ /doi.org/10.1016/S0140-6736(19)32524-3

6. Shirin T, Muraduzzaman AKM, Alam AN, Sultana S, Siddiqua M, Khan MH, Akram A, Sharif AR, Hossain S, Flora MS. Largest dengue outbreak of the decade with high fatality may be due to reemergence of DEN-3 serotype in Dhaka, Bangladesh, necessitating immediate public health attention. New Microbes New Infect. 2019 Feb 16;29:100511. doi: 10.1016/j.nmni.2019.01.007. PMID: 30937172; PMCID: PMC6426716.

7. Islam QT. Dengue Outbreak in 2019. Journal of Medicine. 2020;21(1):1-2. DOI: https://doi.org/10.3329/jom.v $21 \mathrm{i} 1.44092$

8. Kularatnam GA, Jasinge E, Gunasena S, Samaranayake D, Senanayake MP, Wickramasinghe VP. Evaluation of biochemical and haematological changes in dengue fever and dengue hemorrhagic fever in Sri Lankan children: a prospective follow up study. BMC pediatrics. 2019 Dec 1;19(1):87. https://doi.org/10.1186/s12887-019-1451-5

9. Toledo J, George L, Martinez E, Lazaro A, Han WW, Coelho GE, Ranzinger SR, Horstick O. Relevance of noncommunicable comorbidities for the development of the severe forms of dengue: a systematic literature review. PLoS neglected tropical diseases. 2016 Jan;10(1): e0004284. doi: 10.1371/journal.pntd.0004284

10. Pang J, Hsu JP, Yeo TW, Leo YS, Lye DC. Diabetes, cardiac disorders and asthma as risk factors for severe organ involvement among adult dengue patients: A matched casecontrol study. Scientific Reports. 2017 Jan 3;7(1):1-0. DOIhttps://doi.org/10.1038/srep39872

11. Daniel R, Philip AZ. A Study of Clinical Profile of Dengue Fever in Kollam, Kerala, India. Dengue Bulletin. 2005;29:197-202.

12. Ferede G, Tiruneh M, Abate E, Wondimeneh Y, Gadisa E, Howe R, Aseffa A, Tessema B. A study of clinical, hematological, and biochemical profiles of patients with dengue viral infections in Northwest Ethiopia: implications for patient management. BMC infectious diseases. 2018 Dec 1;18(1):616.DOIhttps://doi.org/10.1186/s12879-018$3557-\mathrm{z}$

13. Tewari K, Tewari VV, Mehta R. Clinical and hematological profile of patients with dengue fever at a tertiary care hospital-an observational study. Mediterranean journal of hematology and infectious diseases. : e2018021, DOI: http:/ /dx.doi.org/10.4084/MJHID.2018.021 
14. Alam AS, Sadat SA, Swapan Z, Ahmed AU, Karim MN, Paul HK, Zaman S. Clinical profile of dengue fever in children. Bangladesh Journal of Child Health. 2009;33(2):55-8.

15. Shultana K, Rahman AM, Al Baki A, Khan MS, Deb B, Chowdhury D, Mir AR, Sabrina F, Zaman S, Haque MM. Dengue Infection in Children: Clinical Profile and Outcome in Dhaka City. American Journal of Pediatrics. 2019;5(3):111-5.doi: 10.11648/j.ajp.20190503.16

16. Laul A, Laul P, Merugumala V, Pathak R, Miglani U, Saxena P. Clinical profiles of dengue infection during an outbreak in Northern India. Journal of tropical medicine. 2016;2016.https://doi.org/10.1155/2016/5917934

17. Schexneider KI, Reedy EA. Thrombocytopenia in dengue fever. Current hematology reports. 2005 Mar;4(2):145-8.
18. Deshwal R, Qureshi MI, Singh R. Clinical and laboratory profile of dengue fever. J Assoc Physicians India. 2015 Dec;63(12):30-2.

19. Ojha A, Nandi D, Batra H, Singhal R, Annarapu GK, Bhattacharyya S, Seth T, Dar L, Medigeshi GR, Vrati S, Vikram NK. Platelet activation determines the severity of thrombocytopenia in dengue infection. Scientific reports. 2017 Jan 31;7(1):1-0. https://doi.org/10.1038/srep41697

20. Jyothi P, Metri BC. Correlation of serological markers and platelet count in the diagnosis of Dengue virus infection. Advanced biomedical research. 2015;4:26doi: 10.4103/ 2277-9175.150396

21. Tsai JJ, Chang JS, Chang K, Chen PC, Liu LT, Ho TC, Tan SS, Chien YW, Lo YC, Perng GC. Transient monocytosis subjugates low platelet count in adult dengue patients. Biomedicine Hub. 2017;2(1):1-6. doi:10.1159/000457785. 